\title{
Mechanisms and management of diuretic resistance in congestive heart failure
}

\section{K M De Bruyne}

Postgrad Med J 2003;79:268-271

Diuretic drugs are used almost universally in patients with congestive heart failure, most frequently the potent loop diuretics. Despite their unproven effect on survival, their indisputable efficacy in relieving congestive symptoms makes them first line therapy for most patients. In the treatment of more advanced stages of heart failure diuretics may fail to control salt and water retention despite the use of appropriate doses. Diuretic resistance may be caused by decreased renal function and reduced and delayed peak concentrations of loop diuretics in the tubular fluid, but it can also be observed in the absence of these pharmacokinetic abnormalities. When the effect of a short acting diuretic has worn off, postdiuretic salt retention will occur during the rest of the day. Chronic treatment with a loop diuretic results in compensatory hypertrophy of epithelial cells downstream from the thick ascending limb and consequently its diuretic effect will be blunted. Strategies to overcome diuretic resistance include restriction of sodium intake, changes in dose, changes in timing, and combination diuretic therapy.

\section{Correspondence to: Dr Lode De Bruyne, Department of Cardiology, University Hospital Gent, De Pintelaan 185, 9000 Gent, Belgium; lode_de_bruyne@hotmail.com}

Submitted 1 July 2002

Accepted

10 February 2003
$M$ any of the clinical features of congestive heart failure ( $\mathrm{CHF}$ ) result from retention of sodium and fluid. Although diuretics have not been shown to improve survival in patients with $\mathrm{CHF}$, they do improve symptoms of congestion very effectively. It should also be borne in mind that diuretics have been part of standard CHF therapy in all recent survival trials of $\beta$ blockers, angiotensin converting enzyme inhibitors, and angiotensin II receptor blockers. ${ }^{1}$

Only loop diuretics are effective as single Removal of excessive fluid is usually achieved by a combination of salt restriction and loop diuretics, but in some cases oedema persists despite adequate diuretic therapy. This has been termed diuretic resistance. The prevalence of diuretic resistance in the heart failure population is unknown. In a recent retrospective analysis of 1153 patients with advanced CHF 402 patients had diuretic resistance (defined in this study as requirement of furosemide (frusemide) $>80 \mathrm{mg}$ or bumetanide $>2 \mathrm{mg}$ daily). Diuretic resistance was independently associated with total mortality, sudden death, and pump failure death. ${ }^{3}$ agents in moderate to severe heart failure. ${ }^{2}$

\section{CAUSES OF SODIUM RETENTION IN CONGESTIVE HEART FAILURE}

Baroreceptors on the arterial side of the circulation sense effective arterial blood volume. These receptors are located in the aortic arch, carotid sinus, left ventricle, and afferent renal arterioles. Arterial underfilling can be caused by decreased cardiac output or by arterial vasodilatation and results in activation of several neurohumeral mechanisms that aim to restore effective arterial blood volume by retaining sodium and water. ${ }^{45}$ Activation of the renin-angiotensin system occurs in moderate to severe cardiac failure and after initiation of diuretic therapy. Angiotensin II causes vasoconstriction of both afferent and efferent renal arterioles and stimulates release of aldosterone from the adrenal gland. Increased sympathetic activity is responsible for stimulation of sodium reabsorption in the proximal tubule, renal vasoconstriction, and renin-angiotensin system activation. Increased levels of antidiuretic hormone lead to increased water reabsorption in the collecting duct, thereby predisposing to hyponatraemia.

Most patients with CHF have some degree of renal impairment, due to intrinsic renal disease, some degree of prerenal azotaemia, or a combination of both. Loop diuretics inhibit the reabsorption of sodium, chloride, and potassium in the thick ascending limb of Henle's loop (fig 1). Their site of action is the luminar surface of epithelial cells and therefore they have to reach the urine to be effective. Loop diuretics are firmly bound to serum proteins; they reach the tubular lumen predominantly by active secretion and not by glomerular filtration or passive diffusion. In renal insufficiency secretion of furosemide and other loop diuretics is reduced because of accumulation of endogenic organic anions competing with loop diuretics for the receptor sites of the organic anion transporter. ${ }^{2}$ Higher doses are required to overcome this competitive inhibition and to obtain therapeutic urinary concentrations in heart failure patients with renal impairment. The bioavailability of loop diuretics is unaltered in CHF, but peak urinary concentrations are reduced and tend to occur later, resulting in a less powerful diuretic effect. ${ }^{2}$ This is a second pharmacokinetic mechanism that interferes with a satisfactory diuresis.

When a short acting diuretic like furosemide is administered, it will result in natriuresis as long

Abbreviations: CHF, congestive heart failure; NSAIDs, non-steroidal anti-inflammatory drugs 


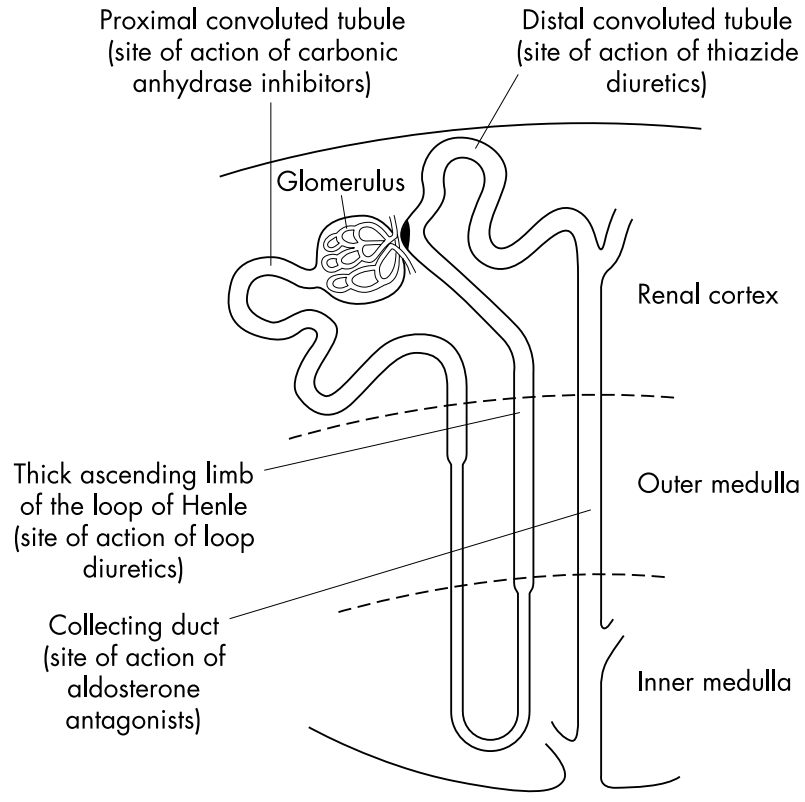

Figure 1 Schematic diagram of the nephron demonstrating the site of action of diuretics.

as its concentration in tubular fluid is high enough to block the $\mathrm{Na}^{+} / \mathrm{K}^{+} / 2 \mathrm{Cl}^{-}$cotransporter. When urinary concentrations decline below the diuretic threshold about six hours later, compensatory sodium retention occurs during the rest of the day. This is called postdiuretic salt retention. If sodium intake is high, postdiuretic salt retention can completely abolish the effect of the diuretic and a negative sodium balance is not achieved. If sodium intake is low, compensatory sodium retention in the postdiuretic phase is incomplete and there is a net loss of sodium.

Chronic administration of loop diuretics results in a diminished natriuretic effect (the "braking phenomenon"). Major determinants of this braking phenomenon are functional and structural adaptations that occur in downstream nephron segments. Studies in rats have shown that chronic administration of a loop diuretic induces hypertrophy and hyperplasia in epithelial cells of the distal convoluted tubule, leading to an increased reabsorption of sodium in this segment, thereby blunting the natriuretic effect and shifting the dose-response curve downward and to the right (fig 2). ${ }^{89}$ Indirect data obtained from a human study are consistent with the data obtained from rats, indicating that these adaptations also occur in humans. ${ }^{10}$

\section{MANAGEMENT OF DIURETIC RESISTANCE}

\section{(A) Rule out non-compliance}

When diuretic resistance is encountered, non-compliance with either salt restriction (sodium intake $<100 \mathrm{mmol} /$ day) or medication intake should first be excluded. As discussed above, postdiuretic salt retention can completely compensate for sodium that is lost during the period in which the diuretic achieves an effective tubular concentration if sodium intake exceeds $100 \mathrm{mmol} /$ day. ${ }^{7}$ Sodium intake can be assessed from measurement of 24 hour salt excretion in the steady state. In subjects already receiving diuretic therapy dietary noncompliance is suspected when daily salt excretion is high ( $>100 \mathrm{mmol} /$ day) without concurrent weight loss. Compliance with diuretic therapy can be assessed by measuring the amount of diuretic in the urine; this may provide useful information to the clinician in a very limited number of cases.

Use of non-steroidal anti-inflammatory drugs (NSAIDs) is a major cause of apparent diuretic resistance. These drugs

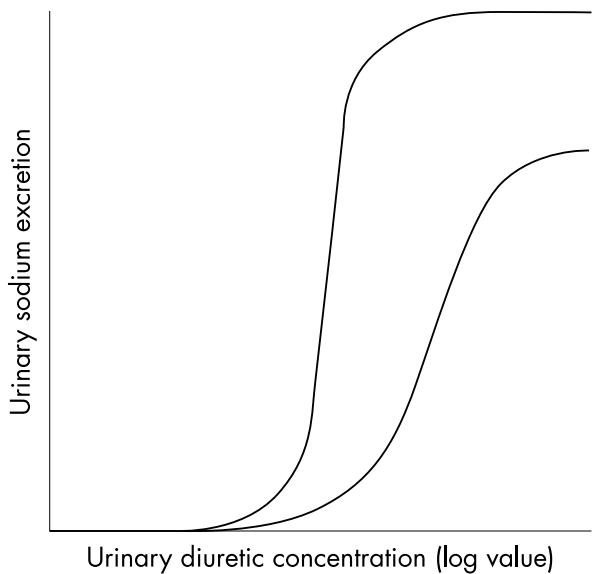

Figure 2 Relationship between renal furosemide excretion rate or urinary diuretic concentration (log value) and excretion of sodium in normal subjects (left curve) and in patients with CHF (right curve).

Box 1: Pharmacokinetic and pharmacodynamic causes of diuretic resistance

- Delayed absorption of the diuretic.

- Reduced secretion of the diuretic into the tubular lumen (its site of action).

- Compensatory retention of sodium after the effective period of the diuretic.

- Hypertrophy and hyperplasia of epithelial cells of the distal convoluted tubule.

interfere with prostaglandin synthesis by inhibiting cyclooxygenase and thereby antagonise the natriuretic response to loop diuretics. Administration of prostaglandin E2 has been shown to restore the natriuretic response to furosemide in indomethacin treated rats. ${ }^{11}$ Consumption of NSAIDs is associated with an increased risk of hospital admission because of heart failure in patients with pre-existing heart failure. ${ }^{12}$

\section{(B) Dose adjustment}

Increasing the dose may be an effective therapeutic strategy because it compensates for the changes in the pharmacokinetics and pharmacodynamics of loop diuretics that occur in patients with CHF. Furosemide is an organic acid that reaches the tubular fluid by secretion via the organic anion transporter of the proximal tubule. Both diminished renal blood flow and reduced activity of the organic anion transporter (due to raised levels of endogenic organic anions) interfere with furosemide secretion in patients with renal impairment. This results in lower concentrations in the renal tubule. Since many patients manifest some degree of renal impairment, increasing the dose will be necessary to deliver appropriate amounts of diuretic to the urinary site of action. Few investigators have studied the use of high dose furosemide in the treatment of refractory CHF. ${ }^{13}{ }^{14}$ Gerlag and van Meijel demonstrated the efficacy and safety of high dose furosemide (250-4000 mg/day, given orally or intravenously) in 35 patients with severe CHF and significantly reduced renal function refractory to conventional diuretic therapy. No significant side effects were noted and weight reduction and relief of symptoms were achieved in all patients. ${ }^{14}$

Since most loop diuretics are short acting, postdiuretic salt retention is an important mechanism contributing to diuretic resistance, particularly when salt intake is not sufficiently restricted. More frequent administration of the diuretic (two to three times a day) overcomes the effect of postdiuretic salt retention by reducing the drug-free interval. 
Urinary concentrations that induce an adequate diuresis in healthy persons may fail to achieve the desired effect in patients with CHF, for dose-response curves are shifted downward and rightward in CHF. ${ }^{15}$ Therefore, it is often necessary to increase the dose of a loop diuretic, even in the absence of pharmacokinetic abnormalities.

Some clinicians consider bumetanide to be more effective than furosemide in patients with CHF because of its better oral bioavailability. Both in normal subjects and in patients with CHF bumetanide has an $80 \%$ bioavailability compared to $40 \%$ for furosemide. ${ }^{16}$ Although bumetanide is 40 times more potent than furosemide on a weight basis, both drugs are equally effective when equipotent doses are administered. ${ }^{17}{ }^{18}$

\section{(C) Intravenous bolus injection or continuous infusion of a loop diuretic}

Impaired absorption of loop diuretics in subjects with CHF results in decreased and delayed peak concentrations in the urine, although the absolute bioavailability does not change significantly when compared with normal subjects. ${ }^{6}{ }^{19}$ A moderate increase in dose or switching to intravenous administration may obviate this problem..$^{13}$

Venous capacitance increases and pulmonary artery pressure decreases within minutes of a bolus infusion of furosemide in patients with congestive symptoms due to acute myocardial infarction or valvular disease. ${ }^{20}$ This finding may explain why patients with pulmonary oedema experience rapid relief of symptoms after administration of intravenous furosemide, even before significant diuresis occurs.

Ototoxicity may occur after rapid intravenous injection of a high dose of a loop diuretic, usually in patients receiving other ototoxic drugs, particularly aminoglycoside antibiotics. Hearing loss and tinnitus are usually transient. ${ }^{21}$

Continuous intravenous infusion of a loop diuretic may be effective when other strategies to overcome diuretic resistance have failed. It will prevent postdiuretic salt retention completely and has been demonstrated to be a safe and effective treatment in patients with CHF refractory to $250 \mathrm{mg}$ furosemide given orally or intravenously. Several controlled studies have compared the efficacy of intermittent intravenous bolus administration of a loop diuretic with continuous infusion in patients with advanced heart failure. ${ }^{22-25}$ The dose of continuous infusions of furosemide ranged from as low as 3 $\mathrm{mg} /$ hour to as high as $200 \mathrm{mg} /$ hour, with most patients receiving 10-20 mg/hour; bumetanide was administered as $0.5 \mathrm{mg}$ bumetanide bolus followed by a continuous infusion of $0.5 \mathrm{mg}$ bumetanide/hour. The same daily dose caused excretion of a higher volume of urine and electrolytes when given as a continuous infusion. The maximal plasma furosemide concentration was significantly lower and this resulted in a reduced risk for ototoxic side effects.

\section{(D) Combination diuretic therapy}

Even patients with refractory heart failure will usually respond to high doses of furosemide administered either orally or as a continuous infusion. However, in some of these patients the use of high dose furosemide does not overcome diuretic resistance.

Several combinations with loop diuretics are possible. Proximal diuretics should be avoided in patients with heart failure because they cause metabolic acidosis. Only one study has been published that reported a successful response to introduction of the potassium-sparing diuretic spironolactone in 13 of 16 heart failure patients refractory to high doses of a loop diuretic. ${ }^{26}$ One patient developed reversible hyperkalaemia $(6.6 \mathrm{mmol} / \mathrm{l})$ and azotaemia due to dehydration. It should be noted that the dose used ( $100 \mathrm{mg}$ once a day) was much higher than the average dose $(25 \mathrm{mg}$ once a day) of spironolactone in the RALES study that produced a survival benefit. ${ }^{27}$ When this high dose is administered, serum potassium levels and hydration status should be monitored during therapy and a maintenance dose of $25 \mathrm{mg}$ should be prescribed after removal of excessive fluid. However, the evidence in favour of spironolactone is rather limited and its use in the treatment of diuretic resistance in CHF is not recommended by the author.

Three studies have assessed the efficacy of combination therapy with thiazide diuretics and loop diuretics in CHF. ${ }^{28-30}$ Thiazide diuretics proved to be highly effective in establishing a diuresis in patients resistant to high doses of loop diuretics. Most patients experienced a significant weight loss and an improvement in functional class when a thiazide diuretic was added to their treatment. Side effects included hypokalaemia, hyponatraemia, dehydration, and renal failure. All patients had a very short life expectancy; failure to respond to thiazide therapy was associated with an even more ominous prognosis. $^{28} 29$

Traditionally metolazone has been used in combination with loop diuretics, although there is no theoretical advantage of one thiazide over another. Addition of $25-100 \mathrm{mg}$ of hydrochlorothiazide, another thiazide diuretic, proved to be very effective in patients with severe CHF and impaired renal function showing diuretic resistance to a daily dose of furosemide of at least $250 \mathrm{mg} \cdot{ }^{30}$ Only one study compared metolazone and another thiazide diuretic in the treatment of severe resistant CHF. Bendrofluazide $10 \mathrm{mg}$ and metolazone $10 \mathrm{mg}$ were equally effective in establishing a diuresis when combined with loop diuretics. ${ }^{29}$ Based on the available data there is no reason to assume that metolazone is superior to other thiazide diuretics.

Thiazide diuretics block the reabsorption of a mere $5 \%-10 \%$ of filtered sodium, whereas loop diuretics are able to block the reabsorption of about $25 \%$ of filtered sodium. Consequently, thiazide diuretics have a weak natriuretic effect and they are ineffective as monotherapy in patients with advanced heart failure. However, when the sodium load in the distal tubule increases chronically, this segment of the nephron can increase its salt transport capacity. This has been demonstrated by Stanton and Kaissling in animal studies. ${ }^{89} \mathrm{~A}$ chronic increase in sodium delivery to (and sodium uptake by) the distal tubule stimulates the transport capacity of this segment by inducing alterations in cell ultrastructure. This mechanism has only been demonstrated indirectly in humans, ${ }^{10}$ but the effectiveness of the addition of a thiazide diuretic to high dose loop diuretic therapy in patients with renal failure or heart failure suggests that similar adaptations occur in humans. ${ }^{31}$ Combining loop and thiazide diuretics in patients with CHF and diuretic resistance is a very elegant and logical treatment option because it takes into account this pathophysiological mechanism.

\section{CONCLUSION}

The vast majority of patients presenting with acute symptoms of fluid overload are responsive to diuretic drugs, at least initially. However, after chronic exposure to loop diuretics, some patients will require increasing doses and eventually develop diuretic resistance. After excluding compliance problems, higher doses and more frequent administration should be tried. Switching to the intravenous route bypasses the gastrointestinal tract and can overcome problems associated with delayed absorption. Continuous intravenous infusion has been shown to be more efficient than intermittent bolus administration. The addition of a thiazide diuretic to treatment with loop diuretics will usually establish a diuresis even in patients not responsive to other diuretic regimens. The mechanism behind this synergistic effect is hypertrophy of cells in the distal collecting tubule (with resulting increases in salt transport capacity) that occurs after chronic high dose loop diuretic therapy. When diuretic resistance has been treated successfully, heart failure treatment should be optimised according to the most recent guidelines in order to reduce mortality. 


\section{Box 2: Practical approach to diuretic resistance}

- Assess compliance with salt restriction and medicine intake. If necessary, measure the amount of salt and diuretic in the urine.

- Discontinue NSAIDs.

- Adjust the dose of the diuretic in patients with renal impairment.

- Switch to intravenous administration to overcome problems associated with impaired absorption.

- As it avoids postdiuretic salt retention, a continuous intravenous infusion of a loop diuretic may succeed where other treatments have failed.

- Combine loop diuretics with other diuretics, preferably a thiazide diuretic.

\section{REFERENCES}

1 Packer M, Cohn JN, Abraham WT. Consensus recommendations for the management of chronic heart failure. Am J Cardiol 1999;83:1A-38.

2 Wilcox CS. Diuretics. In: Brenner BM, Rector FC, eds. The kidney. Philadelphia: WB Saunders, 1996: 2299-330.

3 Neuberg GW, Miller AB, $\mathrm{O}^{\prime}$ Connor $C M$, et al. Diuretic resistance predicts mortality in patients with advanced heart failure. Am Heart 2002;144:31-8.

4 Schrier MW, Abraham WT. Hormones and hemodynamics in heart failure. N Engl J Med 1999;341:577-85

5 Packer M. Pathophysiology of chronic heart failure. Lancet 1992; 340:88-92.

6 Vargo DL, Kramer WG, Black PK, et al. Bioavailability, pharmacokinetics, and pharmacodynamics of torsemide and furosemide in patients with congestive heart failure. Clin Pharmacol Ther 1995;57:601-9.

7 Ellison DH. Diuretic resistance: physiology and therapeutics. Semin Nephrol 1999:19:581-97.

8 Kaissling B, Stanton BA. Adaptation of distal tubule and collecting duct to increased sodium delivery. I. Ultrastructure. Am J Physiol 1988;255:F1256-68

9 Stanton BA, Kaissling B. Adaptation of distal tubule and collecting duct to increased Na delivery. II. Na+ and K+ transport. Am J Physiol 1998;255:F1269-75

10 Loon NR, Wilcox CS, Unwin RJ. Mechanism of impaired natriuretic response to furosemide during prolonged therapy. Kidney Int $1989 ; 36: 682-9$

11 Kirchner KA, Martin CJ, Bower JD. Prostaglandin E2 but not I2 restores furosemide response in indomethacin-treated rats. Am J Physiol 1986;250:F980-5.
12 Feenstra J, Heerdinck ER, Grobbee DE, et al. Association of nonsteroidal anti- inflammatory drugs with first occurrence of heart failure and with relapsing heart failure. Arch Intern Med 2002;162:262-70.

13 Kuchar DL, O'Rourke MF. High dose furosemide in refractory cardiac failure. Eur Heart J 1985;6:954-8.

14 Gerlag PGG, van Meijel JJM. High-dose furosemide in the treatment of refractory congestive heart failure. Arch Intern Med 1988;148:286-91.

15 Brater DC, Chennavasin P, Seiwell R. Furosemide in patients with heart failure: shift in dose-response curves. Clin Pharmacol Ther 1980;28:182-6.

16 Brater DC, Day B, Burdette A, et al. Bumetanide and furosemide in heart failure. Kidney Int 1984;22:183-9.

17 Abrams J. Intramuscular bumetanide and furosemide in congestive heart failure. J Clin Pharmacol 1981;21:673-9.

18 Sagar S, Sharma BK, Sharma PL, et al. A comparative randomized double-blind clinical trial of bumetanide and furosemide in congestive cardiac failure and other edema states. Int $\mathrm{J}$ Clin Pharmacol Ther Toxicol 1984;22:473-8.

19 Vasko MR, Brown-Cartwright D, Knochel JP, et al. Furosemide absorption altered in decompensated congestive heart failure. Ann Intern Med 1985:102:314-18.

20 Raftery EB. Hemodynamic effects of diuretics in heart failure. $\mathrm{Br}$ Heart J 1994;72:44.

21 Ryback LP. Ototoxicity of loop diuretics. Otolaryngol Clin North Am 1993;26:829-44

22 Lahav M, Regev A, Ra'anani P, et al. Intermittent administration of furosemide vs continuous infusion preceded by a loading dose for congestive heart failure. Chest 1992;102:725-31.

23 van Meijel JJ, Smits P, Dormans T, et al. Continuous infusion of furosemide in the treatment of patients with congestive heart failure and diuretic resistance. J Intern Med 1994:235:329-34.

24 Dormans TPJ, van Meijel JJM, Gerlag PGG, et al. Diuretic efficacy of high dose furosemide in severe heart failure: bolus injection versus continuous infusion. J Am Coll Cardiol 1996;28:376-82

25 Ferguson JA, Sundblad KJ, Becker PK, et al. Role of duration of diuretic effect in preventing sodium retention. Clin Pharmacol Ther 1997;62:203-8.

26 van Vliet AA, Donker AJM, Nauta JJP, et al. Spironolactone in congestive heart failure refractory to high-dose loop diuretic and low-dose angiotensin-converting enzyme inhibitor. Am J Cardiol 1993:71:21A-8.

27 Pitt B, Zannad F, Remme WJ, et al. The effect of spironolactone on morbidity and mortality in patients with severe heart failure. N Engl J Med 1999;341:709-17.

28 Kiyingi A, Field M, Pawsey CC, et al. Metolazone in treatment of severe refractory congestive cardiac failure. Lancet 1990;335:29-31.

29 Channer KS, Mclean KA, Lawson-Matthew $P$, et al. Combination diuretic treatment in severe heart failure: a randomised controlled trial. $\mathrm{Br}$ Heart J 1994:71:146-50.

30 Dormans TPJ, Gerlag PGG. Combination of high-dose furosemide and hydrochlorothiazide in the treatment of refractory congestive heart failure.
hy Eur Heart J 1996;17:1867-74.

31 Fliser D, Schröter $M$, Neubeck M, et al. Coadministration of thiazides increases the efficacy of loop diuretics even in patients with advanced renal failure. Kidney Int 1994:46:482-8. 